\title{
ÉTICA Y HUMANITARISMO: DIMENSIONES MORALES DE LA AYUDA HUMANITARIA DESDE LA PERSPECTIVA DE THOMAS POGGE
}

\author{
Laura F. Belli \\ Universidad de Buenos Aires - CONICET, Argentina \\ Silvia Quadrelli \\ Médecins du Monde International
}

Resumen.- El problema de la pobreza global ha alcanzado proporciones gigantescas. Durante los últimos 20 años la cantidad de muertes por causa del hambre y por enfermedades que podrían ser evitadas han ascendido aproximadamente a 250 millones de personas. Thomas Pogge, sostiene que los Estados ricos tienen la responsabilidad de ayudar a aquellos que se encuentran sumidos en la pobreza severa. Esta responsabilidad -que podría entenderse también como un deber moral- resulta del daño, muchas veces evitable, que el orden institucional global actual ha perpetrado sobre Estados pobres. La existencia de la pobreza global, para este autor, constituye una clara violación de los Derechos Humanos.

Palabras clave.- justicia global; pobreza; ayuda humanitaria; acción humanitaria; ética

\begin{abstract}
For the last 20 years the amount of deaths because of hunger and diseases that could be avoided affects approximately 250 million people. Thomas Pogge affirms that the rich countries have the responsibility to help the other countries that are sunk in severe poverty. This responsibility -that could also be understood as moral duty-comes from the damage, often avoidable, that the present global institutional order has perpetrated upon poor States. Global poverty, according to this author, is a clear violation of the human rights, as well as a crime towards humanity.
\end{abstract}

Key words.- global justice; poverty; humanitarian aid; humanitarian action; ethics

La acción humanitaria se despliega en un conjunto diverso de acciones — sean ya nacionales o internacionales-que se dirigen a proveer ayuda a las víctimas de diferentes tipos de desastres (ya sea a aquellas que han sufrido catástrofes naturales o de conflictos armados). Dichas acciones se encuentran orientadas a aliviar el sufrimiento, garantizar la subsistencia, y proteger la dignidad y los derechos fundamentales de estos sujetos. En ciertos casos, también, se intenta además detener el proceso de desestructuración socioeconómica de la comunidad afectada.

La acción humanitaria se encuentra regida por cuatro principios esenciales. Humanidad: con el fin de asegurar un trato humano y digno a quien recibe la asistencia, sean cuales fueren las circunstancias en las cuales se halla inmerso. Independencia: la acción humanitaria debe encontrarse siempre libre de cualquier coerción política, económica, militar o religiosa para lo cual debe asegurarse la independencia financiera y de pensamiento. Imparcialidad: para lograr prestar ayuda en defensa de un espíritu universal y sin ningún tipo de 
discriminación racial, de género, religiosa o ideológico-política. Y, finalmente, neutralidad: que impida tomar partido por alguna de las partes enfrentadas en un conflicto ${ }^{1}$.

En general existe acuerdo en respetar ciertos criterios específicos, como son: la satisfacción de las necesidades básicas; el respeto cultural; la imparcialidad y neutralidad; y la independencia de la ayuda ${ }^{2}$. Muchos de estos principios son recogidos por el Código de Conducta Relativo al Socorro en Casos de Desastre de la Cruz Roja de 1994. Sin embargo, existen diferentes modelos que rigen la ayuda humanitaria de acuerdo con presupuestos teóricos disímiles. Muchos agentes humanitarios inmersos en la posición tradicional, consideran que su tarea responde a cuestiones de caridad. De acuerdo con esta postura, no existe un deber moral de ayuda a los más necesitados, dicha asistencia cuando se ofrece - responde simplemente a motivos caritativos. Esta idea responde a la distinción entre derechos positivos y derechos negativos: las personas poseen un fuerte derecho negativo a la no-interferencia pero no poseen un derecho positivo a ser ayudados. La decisión asistir a los necesitados, entonces, no se desprende de un deber moral, sino de un sentimiento de caridad.

Otra postura, en contraste con la tradicional, supone la existencia de un deber moral de prestar asistencia y aboga a favor de un derecho positivo de ayuda. Esta es la posición defendida, entre otros, por Peter Singer ${ }^{3}$, quien argumenta que nuestros deberes y obligaciones se extienden hasta el punto en que entra en juego el sacrificio de algo que resulte moralmente significativo para nosotros. Sin embargo, como veremos más adelante, si bien esta postura se acerca a la analizada en este trabajo, la misma parece quedarse en un deber "individual", difícil de concebir como una propuesta a nivel global.

Una estrategia final es aquella que apela al deber de rectificación. A diferencia de las anteriores, esta posición se centra en las obligaciones de los Estados y de las Instituciones responsables, en gran parte, por la pobreza mundial. Esta es la perspectiva defendida por Thomas Pogge y que analizaremos a continuación.

\section{El deber de ayudar}

Cuando se hace referencia la deber de prestar ayuda desde la perspectiva de Thomas Pogge, no estamos haciendo referencia a la ayuda humanitaria que se ejerce desde una postura de "generosidad". El tipo de ayuda al que se apela, en cambio, se encuentra estrechamente relacionado con una detracción moral

\footnotetext{
${ }^{1}$ No debe perderse de vista que este principio de neutralidad no implica, sin embargo, que se deba mantener un silencio cómplice en caso de violaciones graves a los Derechos Humanos o al Derecho Internacional Humanitario.

${ }^{2}$ Etxeberria Xabier, Ética De La Acción Humanitaria, Publicaciones Universidad de Deusto, Bilbao, 1999.

3 Singer Peter, "Famine, Affluence and Morality", en Lafollette H (ed), Ethics in Practice, Cambridge, Blackwell, 1997.
} 
de las estructuras sociales vigentes, toda vez que existen desigualdades políticas, de poder y de distribución de bienes. Esta propuesta, que se encarna en un ideal de justicia, se erige a partir de un estándar mínimo de vida que supone, a la vez, la construcción de instituciones que trabajen para satisfacer el cumplimiento de dicho criterio. No basta solamente con mejorar la situación de aquel que recibe nuestra ayuda, sino que se debe pensar también en la transformación del sistema —o los sistemas-que han causado la necesidad de dicha ayuda. Para ello es menester una transformación real de las estructuras de producción y distribución que permitan a los individuos acceder a una real participación política.

El problema fundamental de la perspectiva humanitarista de la justicia comprendida como la ayuda humanitaria que consiste en la repartición de bienes por caridad, sin que se encuentre involucrado ningún tipo de deber moral-, es que si bien intenta mejorar el resultado de una situación injusta, no pretende ejercer acción alguna sobre las estructuras que, en primer lugar, fueron las causantes de la pobreza que ahora intentan paliar.

Pogge señala que no solo poseemos deberes positivos hacia la resolución de la pobreza, sino también deberes negativos que nos compelen a actuar. Si bien concede que la hipótesis defendida por Singer y Henry Shue, entre otros ${ }^{4} 6$ puede ser funcional a la mitigación de la pobreza y los problemas asociados a ésta, cree que el problema es que los mismos argumentos que la apoyan pueden resultar "políticamente oportunos" para quienes son los verdaderos responsables del daño ocasionado, al crear la engañosa sugerencia de que no se posee ningún deber negativo estricto para prevenir el daño que se les están pidiendo atenuar. $Y$ es que, siguiendo a Pogge, no se trata simplemente de prestar nuestra ayuda cuando el costo de hacerlo es relativamente bajo, sino comprender que nos encontramos directa o indirectamente implicados en la producción y perpetuación de las pésimas condiciones en las que viven los habitantes de los Estados más pobres y que, a la vez, nos beneficiamos de las enormes desigualdades sociales que reproducimos injustamente. De este modo, somos claros protagonistas del daño que se inflige a estas poblaciones y es por ello que - de acuerdo con esta perspectiva - entra en juego nuestro deber negativo de no dañar, de no continuar perpetuando el daño que estamos haciendo ${ }^{7}$.

Es claro que la formulación positiva de nuestros deberes es mucho más simple de sustantivar. Sólo es necesario mostrar que aquellos individuos que necesitan de nuestra ayuda se encuentran en la pobreza, que nosotros tenemos condiciones de vida muchísimo mejores que las de ellos y que, además, podríamos aliviar gran parte de su sufrimiento sin por ello perjudicar en lo más mínimo nuestra situación actual. Sin embargo, un segundo problema

\footnotetext{
${ }^{4}$ Ibid.

5 Shue Henry, Basic Rights: Subsistence, Affluence, and U.S.Foreign Policy, Princeton University Press, Princeton, 1980.

${ }^{6}$ Unger Peter, Living High and Letting Die: Our Illusion of Innocence, Oxford University Press, Oxford, 1994.

7 Pogge Thomas, "Real World Justice", The Journal Of Ethics (2005a) 9: 29-535.
} 
de esta perspectiva se relaciona con el hecho de que aquel que acepta la formulación positiva piensa que los argumentos morales que ésta proporciona son débiles y discrecionales, y, por lo tanto, no se siente obligado a promover causas valiosas, especialmente las más costosas o las que involucran a aquellos que nos resultan completamente extraños y con los que no compartimos ningún vínculo de comunidad o de cultura.

La pobreza global, señala Pogge, ejemplifica de un modo claro la desigualdad radical. Sin embargo, la misma a menudo no basta para invocar más que un mero deber positivo. La mayoría de los ciudadanos del occidente desarrollado aceptarían la premisa de que, si no se hiciera absolutamente nada para remediar la pobreza, se estaría violando el deber positivo de beneficencia. Pero, declara Pogge, no parece que estuvieran dispuestos a aceptar que estaríamos violando un deber negativo de justicia. Lo cual es un hecho, según el autor, dado que contribuimos constantemente a la perpetuación de su miseria.

La hipótesis central de Pogge expresa que los ciudadanos de los países desarrollados somos activamente responsables de las condiciones de pobreza y miseria en que viven las personas más vulnerables en los países más pobres.

\section{Responsabilidad y pobreza}

Es claro, para Pogge, que esta pobreza masiva no se debe en lo absoluto a la escasez global. Hoy en día, la línea internacional de la pobreza corresponde a aproximadamente 10 USD mensuales por persona. Las 1.2 mil millones de personas que viven debajo de esta línea de pobreza tienen una renta anual agregada de unos 100 mil millones USD. En claro contraste, las rentas nacionales de los veintitrés países de más alto ingreso de la Organización para la Cooperación y el Desarrollo Económicos (OCDE), con 852 millones de ciudadanos, ascienden por encima de los \$24 trillones de dólares ${ }^{8}$. No parece ilógico pensar que 1.2 mil millones pobres constituyen una desigualdad global tan enorme que valdría la pena duplicar o triplicar sus rentas, aún si fuera a expensas de los países de más alta renta -los cuales apenas notarían la diferencia.

Sería ingenuo pensar que la distribución de la renta y de la abundancia no se encuentra fuertemente influenciada por las instituciones económicas que regulan la distribución de un producto social generado en común. Sin embargo, esto no quita que, quienes vivimos en países desarrollados, no estemos también materialmente implicados en la perpetuación de dichas desigualdades. Pogge concede que es innegable que las instituciones económicas locales desempeñan un papel importante en la reproducción de la pobreza extrema en el mundo en desarrollo. Pero esto no demuestra que las instituciones sociales del mundo desarrollado no están implicadas materialmente en mantener ese juego.

${ }^{8}$ UNDP, Human Development Report, 2002, pp. 165-193. 
Que los efectos de las instituciones domésticas corruptas sean tan nocivos como los son, se debe, a menudo, a que las instituciones globales impiden que los pobres emigren a otros lugares donde puedan llevar una vida decente a partir de su trabajo. Es innegable que una cultura de corrupción se extiende a través del sistema político y económico de la gran mayoría de los países en desarrollo. Pero ¿acaso no tiene importancia el hecho de que la mayoría de los países ricos, hasta hace muy poco tiempo, hayan permitido a sus empresas sobornar a los cargos extranjeros considerándolo en algunos casos, incluso, como un arreglo deducible de impuestos? ${ }^{9}$

La desigualdad radical global hoy en día se encuentra determinada por el orden institucional mundial. Los pobres globales viven en un sistema estatal mundial basado en dominios territoriales internacionalmente reconocidos, interconectados a través de una red global de comercio y diplomacia. Este orden global afecta dramáticamente las circunstancias de los pobres globales a través de inversiones, préstamos, transacciones comerciales, sobornos, ayuda militar, turismo sexual, exportaciones culturales, etc. Con frecuencia, su pura supervivencia depende de manera crucial de nuestras opciones de consumo, las cuales determinan en muchos casos el precio de sus alimentos y también sus oportunidades laborales.

Lo anteriormente dicho no implica, claramente, que seamos los únicos responsables de esta situación. Tampoco implica que la solución consista eliminar la interdependencia global aislando a los diferentes Estados, o grupos de Estados. Pero parece menester adoptar conductas activas acerca del modo previsible en que las reglas que estructuran las relaciones internacionales afectan a la incidencia de la pobreza extrema.

Pogge subraya el hecho de que los países desarrollados, gracias a su inmensa superioridad en fuerza económica y militar, controlan las reglas del mercado y de la política internacional y, por lo tanto, comparten la responsabilidad por sus efectos previsibles. Es por ello que, al apoyar un determinado orden social que sostiene las condiciones a través de las cuales se mantiene una pobreza extrema evitable, nosotros mismos somos responsables del daño que se le está infligiendo a las posiciones más pobres. La ilusión de que los factores domésticos son los únicos determinantes de la pobreza, no hace más que ocultar el verdadero grado de profundidad de la influencia del orden global existente en las economías y políticas locales de los países pobres.

\section{Responsabilidad por la producción de las condiciones que llevan a la pobreza}

Pogge apela también a nuestra responsabilidad en la producción de las condiciones que históricamente han llevado a los pueblos a la pobreza. De acuerdo con el autor, el mundo actual no solo puede ser definido por sus tremendas desigualdades, sino también por el hecho de que el "punto de

${ }^{9}$ Pogge Thomas W., World Poverty and Human Rights, Polity Press, Cambridge, 2002. 
partida social" de los que se encuentran en peor situación y de aquellos que se encuentran mejor proviene de un único proceso histórico cargado de culpas morales $^{10}$. La mayor parte de las desigualdades entre las naciones del mundo tienen su origen en el período colonial en que, los hoy países desarrollados, definieron el futuro de las hoy naciones en desarrollo. Período caracterizado por el comercio irrestricto, la destrucción de las instituciones políticas de los pueblos colonizados, de sus culturas y la apropiación ilimitada de sus recursos naturales.

Pogge sostiene que el orden institucional actual es violatorio de los Derechos Humanos en tanto impide previsiblemente el acceso a cientos de miles de personas de los países pobres al pleno disfrute de dichos derechos básicos. Dicha restricción no debe mantenerse en la continuación de las posiciones sociales de partida, porque no se debe permitir que una historia tan profundamente corrupta desde el punto de vista moral continúe reproduciendo una desigualdad tan radical. Por lo que concluye que, en tanto y en cuanto los ciudadanos más privilegiados de los países ricos contribuyan sustancialmente a mantener el actual orden económico - sea de su propia sociedad o de la sociedad global-, puede decirse de ellos que poseen fuertes razones morales para mitigar las consecuencias de la existencia de dicho orden que ellos mismos sostienen.

Si se acepta la propuesta de Pogge, no solamente el deber moral de ayudar es imperativo (ya que se entiende como el deber negativo de mitigar el daño, de no dañar) sino que, además, se deberá reconocer que las catástrofes a las que se encuentran sometidas las poblaciones vulnerables (la pobreza, la violencia y sus consecuencias) poseen causas identificables y, por ende, responsables identificables.

\section{La perspectiva de Pogge en la ayuda humanitaria}

Los actores humanitarios que aceptan esta perspectiva ético-política -a pesar de existir muchos posibles matices - no podrán, claramente, limitarse solo a las acciones de ayuda humanitaria. En tanto acepten que la pobreza y sus consecuencias se relacionan estrechamente con un orden económico y político mundial y que son (al menos parcialmente) evitables, su tarea no puede limitarse simplemente a alguna forma de ayuda, sino que requiere de un accionar permanente y comprometido para lograr cambiar el orden económico y político global actual que impone un orden social injusto a las poblaciones más vulnerables. Por ello estos actores (que incluyen fundamentalmente a las llamadas organizaciones de "nuevo orden" y a gran parte del movimiento de los French Doctors) saben que —-más que la ayuda humanitaria - su tarea es la acción humanitaria con su componente de testimonio, denuncia y advocacy.

\footnotetext{
10 Pogge, "Recognized and Violated by International Law: The Human Rights of the Global Poor", Leiden Journal of International Law, Vol. 18, No. 4, 2005b, pp. 717-745.
} 
Las organizaciones que responden a este modelo, son organizaciones profundamente políticas que militan activamente para interpelar a quienes consideran los responsables de un orden social injusto, tanto a nivel de las elites locales, como a nivel de los países desarrollados que regulan de múltiples maneras el orden económico y social global. Para estas organizaciones la ayuda es esencial, pero insuficiente. La pobreza no sólo debe ser mitigada por quienes más tienen, sino que las relaciones institucionales globales que llevan a que exista esa pobreza extrema deben ser también modificadas. Apelan entonces a sus donantes no solamente para que contribuyan desde la responsabilidad del deber positivo, sino para que además actúen para que las sociedades que sostienen y defienden dejen de dañar a las poblaciones más vulnerables.

\section{Conclusiones}

La posición sobre la noción de responsabilidad moral que hemos expuesto -la cual es objeto de calurosos debates en el campo del derecho, de la bioética y de la filosofía política-, no agota el amplio espectro de argumentos sobre justicia distributiva, Derechos Humanos y responsabilidad de los ciudadanos globales más aventajados hacia los más pobres. Pero creemos que logra ejemplificar claramente una posición paradigmática en su relación con la fundamentación de un buen quehacer humanitario.

La acción humanitaria entronca su esencia en la dignidad de las víctimas vulneradas, lo que queda referido y recogido normativamente por medio de los derechos. La preocupación creciente de los humanitarios respecto a la relevancia de la protección de los Derechos Humanos, proviene justamente de la doctrina ahora dominante que entiende que no es posible resolver necesidades humanitarias en términos de protección y ayuda mientras se desatienden las causas de estas necesidades en el primer lugar.

Durante los últimos años, las consecuencias desastrosas de la acción humanitaria en muchas de las crisis recientes han movilizado a muchos sectores del espacio humanitario hacia este "nuevo humanitarismo" de creciente politización y de toma de posiciones políticas en los distintos conflictos $\mathrm{y}$, fundamentalmente, con una perspectiva mucho más orientada hacia los Derechos Humanos ${ }^{11}$. La mayor parte de las acciones humanitarias se relacionan con escenarios de conflictos, tristemente privilegiados para la violación - a veces sistemática- de los derechos sociales o económicos, para la manifestación más cruda de la intolerancia étnica, religiosa o política. Estas realidades son más contundentes en los conflictos armados, dado que son los más virulentos. Basta recordar que en Bosnia-Herzegovina, durante cinco años, 4 millones de personas fueron asistidas por agencias internacionales y ONGs, sin que por ello se impidiera la limpieza étnica o las constantes violaciones a las mujeres.

${ }^{11}$ Fox, Fiona. "The New Humanitarianism: Does It Provide a Moral Banner for the 21st Century?", Disasters. 25:4. 2001, pp. 275-289. 
Pero no sólo las guerras son escenario propicio para estos hechos. En las catástrofes naturales, las causas estructurales asociadas a la violación de los derechos sociales y económicos son determinantes directos de la magnitud del daño. Los pobres, por su existencia marginal en territorios marginales, son afectados inevitablemente en forma desproporcionada por terremotos, inundaciones $u$ otros desastres naturales. El Huracán Mitch fue una de las principales demostraciones de las devastadoras consecuencias de las profundas disparidades entre pobres y ricos en América central.

Numerosas organizaciones humanitarias han descubierto que ayudar a víctimas humanitarias debe ser más que un esfuerzo paliativo y que debe, también, incluir el desarrollo por el respeto por los Derechos Humanos. Hoy en día no alcanza con la provisión de ayuda temporaria, sino que se requiere de respuestas globales que intenten salvaguardar la integridad y la dignidad humanas asegurándole a todas y cada una de las víctimas su derecho a la vida y la libertad y protegiéndolas del tratamiento discriminatorio, humillante 0 inhumano, incluyendo la pobreza.

Esta ética básica de respeto y promoción de los Derechos Humanos debe ser común a todos los actores humanitarios. El respeto y la vigilancia de las normas impuestas por el derecho internacional vigente debe ser un componente ineludible de la acción de todos los actores humanitarios. El difícil ejercicio de la tolerancia acerca de las diferentes concepciones de la ayuda humanitaria es uno más de los muchos y complicados desafíos del espacio humanitario en estos tiempos de necesario respeto a la diversidad cultural.

Parece innegable el hecho de que los ciudadanos de los países ricos se encuentran potencialmente implicados en los horrores que muchos sufren en los —así llamados - países menos desarrollados. Las diferentes concepciones de ayuda humanitaria difieren en sus criterios específicos de la justicia global, sin embargo, convienen en que lo esencial consiste en determinar cuán bien funciona el orden institucional global actual, comparado con las posibles alternativas, teniendo en cuenta — desde una perspectiva moral- los intereses humanos fundamentales que se hallan en juego.

Los ciudadanos de los países desarrollados somos responsables de regímenes globales tales como el sistema de comercio global, así como también de las diversas intervenciones militares. Estos arreglos institucionales afectan a seres humanos a lo largo y a lo ancho del mundo. Quizás desarrollos alternativos de la globalización hubieran arrojado diferencias en relación con la cantidad de violencia, opresión, y pobreza extrema que los caminos tomados han generado. $Y$ es por ello que la necesidad de una reforma institucional global se desprende de la posibilidad de lograr en el futuro - de modo efectivo- el cumplimiento y el respeto por los Derechos Humanos. La importancia de esta perspectiva de la responsabilidad global trasciende los límites de la filosofía teórica: insta a cada ciudadano a evaluar sus opciones y su responsabilidad en la elección y el apoyo de factores institucionales globales, analizando las causas de la pobreza y del hambre trasvasan las fronteras. 


\section{Bibliografía}

(1991) Alegre Marcelo, "Extreme Poverty in a Wealthy World: What Justice Demands Today", en Barry, B., Liberty and Justice: Essays in Political Theory, Oxford, Clarendon Press.

(1974) Campbell Tom, "Humanity Before Justice", British Journal of Political Science.

Código de conducta relativo al socorro en casos de desastre para el Movimiento Internacional de la Cruz Roja y de la Media Luna Roja y las Organizaciones No Gubernamentales http://www.cruzroja.org/pvh/redicom/docs/DocReferencia/Policas/CConducta.pd f

(1996) Consejo de la UE, Reglamento del Consejo (EC) $n^{0} 1257 / 96$ de 20 de junio de 1996 sobre la ayuda humanitaria, Official Journal $n^{\circ}$ L 163, 02/07/1996 P. 0001-0006

(1997) Derrida Jacques , Dufourmantelle Anne De l'hospitalité, Paris, CalmannLévy.

(1995) Dower Nigel, "La pobreza en el mundo", en Peter Singer (ed.), Compendio de Ética, Madrid, Alianza Editorial.

(1977) Dworkin Ronald, "Justice and Rights", en Taking Rights Seriously, London, Duckworth.

(2005) Estébanez Pilar, Medicina humanitaria, Madrid, Díaz de santos.

(1999) Etxeberria Xabier, Ética De La Acción Humanitaria, Bilbao, Publicaciones Universidad de Deusto.

(2001) Fox, Fiona. "The New Humanitarianism: Does It Provide a Moral Banner for the 21st Century?", Disasters. 25:4, pp. 275-289.

(1988) Goodin, R., "What is So Special About our Fellow Countrymen?", Ethics, 98, 4, , pp. 663-86.

(1995) Habermas Jürgen, "Reconciliation through the public use of reason: remarks on John Rawls's political liberalism", The Journal of Philosophy, vol. XCll, n3.

(1990) Levinas Emmanuel, Totalité et infini. Essai sur l'exteriorité, Paris, Le Livre de Poche, Biblio Essais, Nro. 4120.

(2007) Luna Florencia, "Pobreza en el mundo: obligaciones individuales e institucionales y Derechos Humanos", Revista latinoamericana de filosofía, Vol. 33, n 2, pp. 293-314. 
(1977) Nagel T, "Poverty and Food: Why Charity Is Not Enough" in Peter Brown \& Henry Shue (Eds), Food Policy: The Responsibility of the United States in Life and Death Choices, New York, Free Press.

(1974) Nozick, Robert, Anarchy, State, and Utopia, New York, Basic Books.

(1986) O'Neill, O., Faces of Hunger: An Essay on Poverty, Justice and Development, London, Allen \& Unwin.

(2001) Pogge T., "Eradicating Systemic Poverty: brief for a global resources dividend", Journal of Human Development, v. 2, n1, pp. 59-77.

(2002) Pogge Thomas W., World Poverty and Human Rights, Cambridge, Polity Press.

(2005a) "Real World Justice", The Journal Of Ethics 9: 29-535.

, (2005b) "Recognized and Violated by International Law: The Human Rights of the Global Poor", Leiden Journal of International Law, Vol. 18, No. 4, pp. 717-745.

_, (2002) "Responsibilities for Poverty-Related III Health", Ethics \& International Affairs, Vol. 16.

(1971) Rawls, John, A Theory of Justice, Cambridge-Mass., Harvard University Press.

(2006) Risse Mathias, "Do We Owe the Global Poor Assistance or Rectification?", Ethics \& International Affairs, 19 Issue 1, pp. 9-18.

(1980) Shue Henry, Basic Rights: Subsistence, Affluence, and U.S.Foreign Policy, Princeton, Princeton University Press.

(2004) Singer, P., "Outsiders: our obligations to those beyond our borders", en Chatterjee, D. (ed.) The Ethics of Assistance: Morality and the Distant Needy, Cambridge, Cambridge University Press.

, (1997) "Famine, Affluence and Morality", en Lafollette H (ed), Ethics in Practice, Cambridge, Blackwell.

(1994) Unger Peter, Living High and Letting Die: Our Illusion of Innocence, Oxford, Oxford University Press.

(2002) UNDP, Human Development Report, pp. 165, 193. 